\title{
Optical Resonators based on Casimir Forces -INVITED
}

\author{
Sol Carretero-Palacios ${ }^{1,2, *}$, Victoria Esteso ${ }^{1}$, and Hernán Míguez ${ }^{1}$ \\ ${ }^{1}$ Institute of Materials Science of Seville, Consejo Superior de Investigaciones Científícas (CSIC)-Universidad de Sevilla (US), Américo \\ Vespucio 49, 41092 Seville, Spain \\ ${ }^{2}$ Departamento de Física de Materiales, Universidad Autónoma de Madrid, 28049 Madrid, Spain
}

\begin{abstract}
The work here presented demonstrates theoretically that it is possible to create optical resonators based on levitation properties of thin films subjected to repulsive Casimir-Lifshitz forces. Our optical cavity design is made up of commonly found materials, such as silicon oxide, polystyrene or gold, with glycerol as a mediating medium, which supports high Q-factor optical modes at visible frequencies. The balance between flotation and repulsive Casimir-Lifshitz forces in the system allows the fine-tuning of the optical cavity thickness and hence its modes. Finally, we show that well-defined spectral features in the reflectivity allows by indirect means, an accurate prediction of the estimated equilibrium distance at which some part of the optical cavity arrangement levitates.
\end{abstract}

\section{INTRODUCTION}

Recently, in an elegant cutting-edge experiment [1], repulsive $F_{(C-L)}$ have been demonstrated in levitating metallic plates immersed in ethanol through a fashionable stratification of the inner dielectric media, elucidating the quantum trapping distance $\left(d_{e q}\right)$ indirectly by spectroscopic techniques.

Here, we examine theoretically high Q-factor optical resonators formed by a levitating bilayer plate facing a coated planar substrate, all immersed in an appropriate liquid under the influence of repulsive $F_{(C-L)}$ and buoyancy [2]. We show that well-defined spectral features in the reflectivity (identified with high Q-factor optical resonators) allows an accurate prediction of $d_{e q}$ by indirect means. Effects related to repulsive electrostatic forces are also evaluated. Specifically, optical resonators supporting high Q-factor optical modes at visible frequencies are formed by a gold (Au)-polystyrene (PS) bilayer drown in glycerol over an $\mathrm{Au}$ coated silicon dioxide $\left(\mathrm{SiO}_{2}\right)$ substrate (see Figure 1). The selected materials display remarkable optical quality, fine spectral reflectivity features, experimentally manageable thicknesses, and unlike alcohols, stable dissipation properties. Importantly, the relation of the dielectric permittivities gives rise to repulsive Casimir forces able to balance buoyancy.

\section{Results and Discussion}

$F_{(C-L)}$ is calculated employing the Lifshitz's theoretical formalism expressed for an arbitrary layered system [3], whereas the Poisson-Boltzmann methodology is applied to evaluate the effect of double layer forces [4,5]. Reflectance spectra are calculated employing the Transfer
Matrix Method formalism [6,7]. Dielectric permittivities of all materials needed in the calculations are extracted from diverse references, in some cases employing oscillator models directly evaluated in Matsubara frequencies.

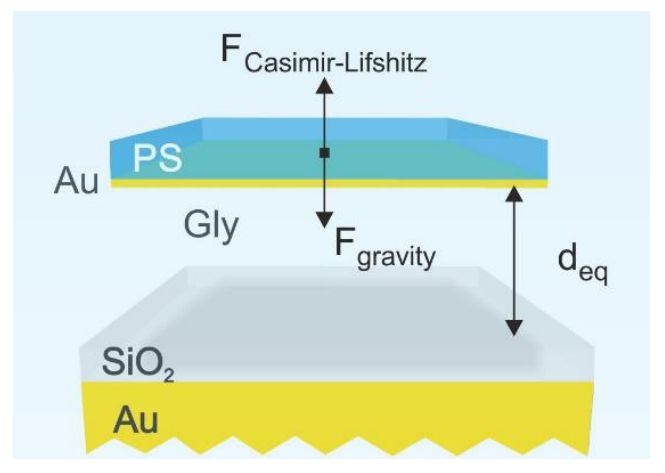

Fig. 1. Scheme of the optical cavity formed by a self-standing thin film made up of gold (Au) and polystyrene (PS) immersed in glycerol over a silica $\left(\mathrm{SiO}_{2}\right)$ coated gold substrate. Layer thicknesses range from tens to several hundreds of nanometers. The equilibrium distance at which Casimir-Lifshitz and gravity forces are balanced results in $d_{e q}$ of few hundreds of nanometers.

Figure 2 shows a representative example of the equilibrium distances at which golden bilayer plates levitate as a function of the PS thickness building the optical resonator. For the set of parameters here considered, these can be finely tuned within $d_{e q} \in[180-$ 375] nm by enhancing $F_{b}$ with the PS thickness.

* Corresponding author: sol.carretero@uam.es 


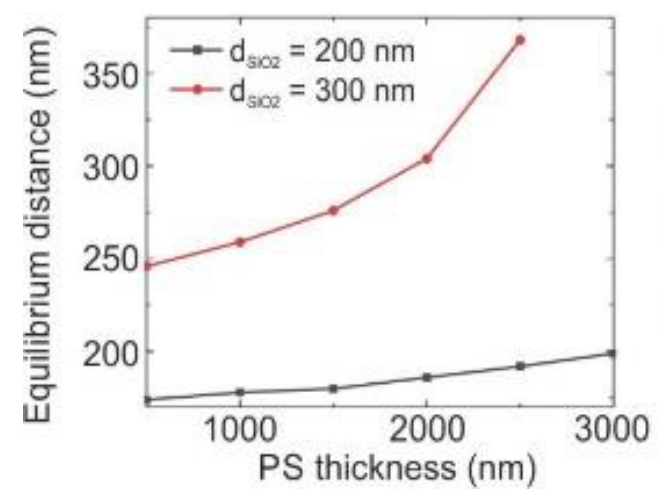

Fig. 2. Equilibrium distance as a function of the PS thickness slab in the levitating bilayer, with and $d_{A u}=30 \mathrm{~nm}$ fixed and setting $d_{\mathrm{SiO}_{2}}=200 \mathrm{~nm}$ (in back) and $300 \mathrm{~nm}$ (in red).
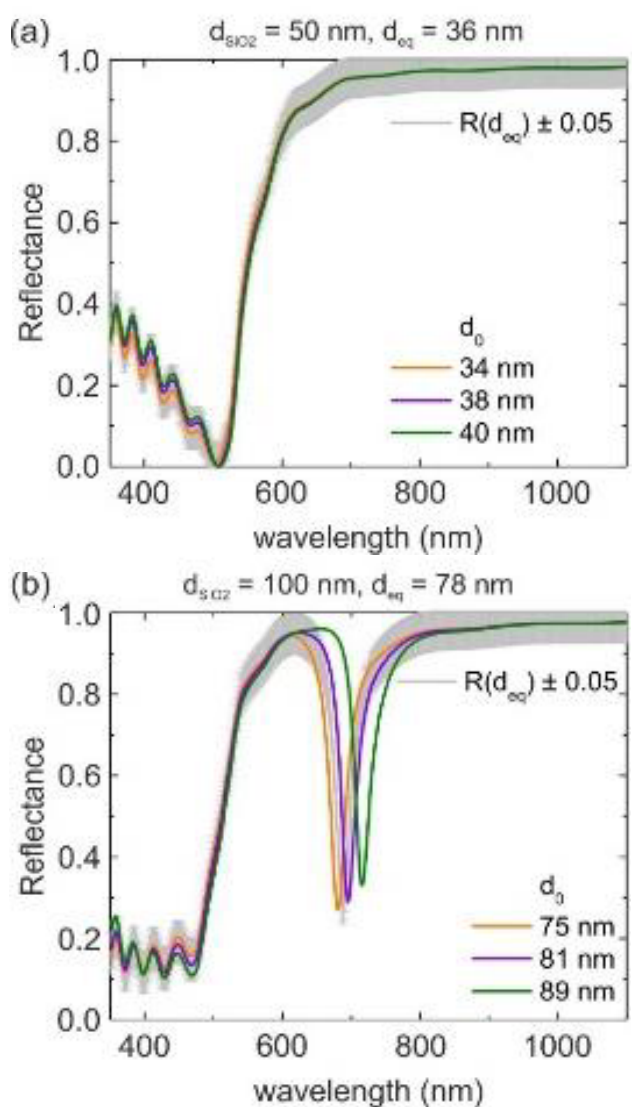

Fig. 3. (a) Reflectance of a system displaying levitation with $d_{P S}=1500 \mathrm{~nm}, d_{A u}=30 \mathrm{~nm}, d_{\text {glycerol }}=d_{e q}=36 \mathrm{~nm}$, and $d_{\mathrm{SiO}_{2}}=50 \mathrm{~nm}$, and an assumed error in the reflectance of \pm 0.05 (in grey). For the same set of parameters, but separation distances $d_{\text {glycerol }}=d_{0}$ of 34,38 and $40 \mathrm{~nm}$, results are shown in orange, purple and green colors. These last systems do not yield levitation. (b) Results for the same set of parameters as in panel (a), but considering $d_{\text {SiO }_{2}}=100 \mathrm{~nm}$, thus, $d_{\text {glycerol }}=$ $d_{e q}=78 \mathrm{~nm}$, and assuming an error of \pm 0.05 (in grey). Also, for separation distances of $d_{\text {glycerol }}=d_{0}$ of 75, 81 and $89 \mathrm{~nm}$, results are shown in orange, purple and green colors. These last systems do not display levitation.

Figure 3 shows with two illustrative examples the need of having well-defined narrow spectral features to accurately estimate $d_{e q}$, and therefore, $F_{(C-L)}$. Both panels display high reflectance bands over a wide spectral range and, as the Q-factor of the optical cavity increases (ranging from 25 to 164 with the cavity size), narrower minima that rise in number appear at distinct wavelengths. For two $\mathrm{SiO}_{2}$ coating thicknesses, $50 \mathrm{~nm}$ (a) and $100 \mathrm{~nm}$ (b), reflectance spectra of levitating films are shown in grey color. A deviation of \pm 0.05 in the reflectance response arising from diverse error sources (such as variations in the estimation of film thicknesses, imperfect parallel alignment, surface roughness, etc) is also assumed. The spectral response of similar systems located close to the equilibrium distance (but without displaying levitation) are also depicted. Notably, for thin $\mathrm{SiO}_{2}$ coating thicknesses (panel (a)), reflectance spectra of systems placed at $d_{0}=34,38$ and $40 \mathrm{~nm}$, match the estimated \pm 0.05 reflectance error. This means that in a possible experiment based on this approach, any gap distance within the range $d_{0} \in$ [34-40] nm could be inaccurately identified to levitation arising from just the balance of Casimir-Lifshitz forces and buoyancy, neglecting for instance the possible effect of additional forces in the system. In high Q-factor optical cavities (panel (b)), $d_{e q}$ can be precisely determined. In this case, narrow spectral minimum corresponds to a single set of parameters, while the spectral response of bilayers located at $d_{0}=75,81$, and $89 \mathrm{~nm}$ fall outside the assumed \pm 0.05 error.

\section{Conclusions}

We have shown that high Q-factor optical resonators allows determining unambiguously, by spectroscopic techniques, the equilibrium distance of a levitating bilayer over a planar substrate due to the balance of repulsive $F_{(C-L)}$ and buoyancy. The selected choice of materials, namely $\mathrm{SiO}_{2}$, PS, Au and glycerol, display decisive characteristics, such as repulsive $F_{(C-L)}$, adequate densities to balance such dispersion force at measurable nanoscale distances, excellent optical quality and manageable experimental fabrication. Besides, glycerol presents stable properties in contrast to alcohols suffering from evaporation.

\section{References}

1. A. Zhao, R.; Li, L.; Yang, S.; Bao, W.; Xia, Y.; Ashby, P.; Wang, Y.; Zhang, X. Science, 2019, 364 (6444), 984-987

2. Esteso, V.; Carretero-Palacios, S. ; Míguez, H. J. Phys. Chem. Lett. 2019, 10, 5856-5860

3. Dzyaloshinskii, I.E.; Lifshitz, E.M.; and Pitaevskii, L.P. Adv. Phys. 1961, 10 (38), 165-209

4. Cappella, B.; Dietler, G. Surface Science Reports, 1999, 34, 1-104

5. Parsegian, V. A.; Gingell, D. Biophys. J., 1972, 12, 1192

6. Thompson, R. C. Optical Waves in Layered Media. J. Mod. Opt. 1990.

7. Hecht, E. Hecht Optics. Addison Wesley 1998. 